\title{
Probabilistic model for pressure vessel reliability incorporating fracture mechanics and nondestructive examination
}

\author{
David M. Tow and Walter G. Reuter \\ Idaho National Engineering and Environmental Laboratory \\ P.O. Box 1625, Idaho Falls, ID 83415-2209 \\ ABSTRACT
}

\begin{abstract}
A probabilistic model has been developed for predicting the reliability of structures based on fracture mechanics and the results of nondestructive examination (NDE). The distinctive feature of this model is the way in which inspection results and the probability of detection (POD) curve are used to calculate a probability density function (PDF) for the number of flaws and the distribution of those flaws among the various size ranges. In combination with a probabilistic fracture mechanics model, this density function is used to estimate the probability of failure (POF) of a structure in which flaws have been detected by NDE. The model is useful for parametric studies of inspection techniques and material characteristics.
\end{abstract}

Keywords: probabilistic fracture mechanics, nondestructive examination, structural reliability

\section{INTRODUCTION}

The key issue addressed in this paper is how to include inspection results in a probabilistic model of the reliability of a structure. Probabilistic fracture mechanics can be used to calculate the probability of failure of a structure due to a single flaw or numerous flaws when the nominal level of applied stress is known. ${ }^{1}$ The random variables in such models are typically material properties, such as fracture toughness, flaw characteristics, such as size and orientation, and values for the applied stress.

When the actual number of the flaws is uncertain the model must be modified to accommodate variability in the flaw population. A method of modeling flaw population as a random variable and combining this model with a probabilistic fracture mechanics model is presented here. A probability density function for the number of flaws and the distribution of those flaws among the various size ranges is computed using NDE results and the POD curve of the inspection technique employed. This PDF is then used to estimate mean probability of failure.

A very simple probabilistic fracture mechanics model is employed for this work. Only variability in the applied stress is considered; all material properties are considered deterministic. The only type of flaws considered are surface cracks with constant aspect ratio. This simplified model is sufficient to demonstrate some of the more important characteristics of the modeling approach.

\section{FLAW POPULATION PROBABILITY DENSITY FUNCTION}

The issue in question is how to estimate the actual number of flaws and their sizes when imperfect NDE techniques are employed to detect them. It is assumed that the POD versus size curve of the NDE technique is known. It is also assumed that flaws can be grouped by size into a finite number of bins. For example, flaws with through-wall thickness fractions of less than .1 might be assigned to the first bin. The next bin might include flaws with through-wall thickness fractions greater than .1 but less than .2 , and so fourth.

A single estimate of the number of flaws in each size bin is not particularly useful if the goal is to estimate statistical moments, such as the POF. A probability density function is desired, which we derive here. To begin, we assume that the

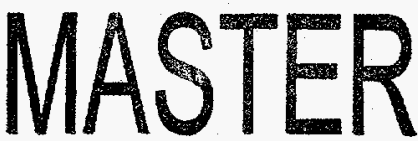




\section{DISCLAIMER}

This report was prepared as an account of work sponsored by an agency of the United States Government. Neither the United States Government nor any agency thereof, nor any of their employees, makes any warranty, express or implied, or assumes any legal liability or responsibility for the accuracy, completeness, or usefulness of any information, apparatus, product, or process disclosed, or represents that its use would not infringe privately owned rights. Reference herein to any specific commercial product, process, or service by trade name, trademark, manufacturer, or otherwise does not necessarily constitute or imply its endorsement, recommendation, or favoring by the United States Government or any agency thereof. The views and opinions of authors expressed herein do not necessarily state or reflect those of the United States Government or any agency thereof. 


\section{DISCLAIMER}

Portions of this document may be illegible electronic image products. Images are produced from the best available original document. 
number of flaw size bins is $R$. Then for $i=1, . ., R$, the probability of there being $m_{i}$ flaws in the $i$-th bin, given that $n_{i}$ flaws have been detected, is denoted:

$$
P_{M \mid N}(m \mid n) \equiv P_{M_{1}, M_{2}, \ldots, M_{R} \mid N_{1}, N_{2}, \ldots, N_{R}}\left(m_{1}, m_{2}, \ldots, m_{R} \mid n_{1}, n_{2}, \ldots, n_{R}\right)
$$

By Bayes' rule

$$
P_{M \mid N}(m \mid n)=\frac{P_{N \mid M}(n \mid m) P_{M}(m)}{P_{N}(n)}
$$

The density function $P_{N M}$ is the probability of $n_{i}$ flaws being detected in the $i$-th bin, given that $m_{i}$ flaws actually exist, for $i=1, \ldots, R$. Because the number of flaws detected in a bin is unrelated to the number of flaws detected in any other bin the PDF is separable as follows:

$$
P_{N \mid M}(\boldsymbol{n} \mid \boldsymbol{m})=\prod_{r=1}^{R} P_{N, \mid M_{r}}\left(n_{r} \mid m_{r}\right)
$$

If the POD for flaws in the $i$-th bin is $p_{i}$, then each term in the product assumes a familiar binomial form, and $P_{M M}$ becomes:

$$
P_{N \mid M}(\boldsymbol{n} \mid \boldsymbol{m})=\prod_{r=1}^{R} \frac{m_{r} !}{n_{r} !\left(m_{r}-n_{r}\right) !} p_{r}^{n_{r}}\left(1-p_{r}\right)^{m_{r}-n_{r}} \equiv \prod_{r=1}^{R}\left(\begin{array}{l}
m_{r} \\
n_{r}
\end{array}\right) p_{r}^{n_{r}}\left(1-p_{r}\right)^{m_{r}-n_{r}}
$$

The density function $P_{M}$ is the unconditional distribution of flaws in the $R$ bins. A related PDF is $P_{M \mid M_{\text {Tor }}}$, the distribution of flaws in the $R$ bins, assuming that the total number of flaws is fixed, i.e. assuming $m_{\text {TOT }}=\sum_{r=1}^{R} m_{r}$ is held constant. If $d_{i}$ is the probability that a flaw will be located in a the $i$-th bin (rather than in any of the remaining $R-1$ bins) then $P_{M M M_{T O T}}$ assumes the familiar multinomial form:

$$
P_{M \mid M_{T O T}}\left(\boldsymbol{m} \mid \boldsymbol{m}_{T O T}\right)=\frac{\left(\sum_{r=1}^{R} m_{r}\right) !}{\prod_{r=1}^{R} m_{r} !} \prod_{r=1}^{R} d_{r}^{m_{r}}
$$

For surface cracks, the $d$ vector is referred to as the crack depth distribution. Typical crack depth distributions are exponential ${ }^{2}$ or lognormal. ${ }^{3}$ If we make the assumption that the total number of flaws, $m_{T O T}$, is uniformly distributed over the range 0 to some value $m_{M A X}$, then we derive the expression for $P_{M}$ :

$$
P_{M}(m)=\frac{1}{m_{\max }+1} \frac{\left(\sum_{r=1}^{R} m_{r}\right) !}{\prod_{r=1}^{R} m_{r} !} \prod_{r=1}^{R} d_{r}^{m_{r}}
$$

The assumption that the total number of flaws is uniformly distributed is not essential, but it does simplify the calculations. It is a conservative assumption in the sense that the density function $P_{N M}$ will be less sharply peaked because of it. 
The remaining term to be identified in equation (1) is $P_{N}$. From probability theory we have:

$$
P_{N}(\boldsymbol{n})=\sum_{m_{1}} \sum_{m_{2}} \ldots \sum_{m_{R}} P_{N, M}(\boldsymbol{n}, \boldsymbol{m})=\sum_{m_{l}} \sum_{m_{2}} \ldots \sum_{m_{R}} P_{M M}(\boldsymbol{n} \mid \boldsymbol{m}) P_{M}(\boldsymbol{m})
$$

Where the summations are over all values of $m_{i}, i=1, \ldots, R$, for which $\sum_{r=1}^{R} m_{r} \leq m_{M A X}$ and $m_{i} \geq n_{i}$. So the denominator of equation (1) becomes:

$$
P_{N}(\boldsymbol{n})=\sum_{m_{1}} \sum_{m_{2}} \ldots \sum_{m_{R}}\left\{\left[\prod_{r=1}^{R}\left(\begin{array}{l}
m_{r} \\
n_{r}
\end{array}\right) p_{r}^{n_{r}}\left(1-p_{r}\right)^{m_{r}-n_{r}}\right]\left[\frac{1}{m_{\max }+1} \frac{\left(\sum_{r=1}^{R} m_{r}\right) !}{\prod_{r=1}^{R} m_{r} !} \prod_{r=1}^{R} d_{r}^{m_{r}}\right]\right\}
$$

Remembering that the vector $\boldsymbol{n}$ is the number of flaws detected in each flaw size range, it is clear that $P_{N}$ assumes a constant value once the NDE results have been obtained. Finally, we arrive at an equation for the probability that a certain population distribution of flaws exists, given that a particular distribution of flaws has been detected:

$$
P_{M \mid N}(\boldsymbol{m} \mid \boldsymbol{n})=\frac{1}{P_{N}(\boldsymbol{n})}\left[\prod_{r=1}^{R}\left(\begin{array}{l}
m_{r} \\
n_{r}
\end{array}\right) p_{r}^{n_{r}}\left(1-p_{r}\right)^{m_{r}-n_{r}}\right]\left[\frac{1}{m_{\max }+1} \frac{\left(\sum_{r=1}^{R} m_{r}\right) !}{\prod_{r=1}^{R} m_{r} !} \prod_{r=1}^{R} d_{r}^{m_{r}}\right]
$$

The calculation of the mean probability of failure using this density function will now be discussed. If $f_{i}$ is the POF due to one flaw in the $i$-th size range, then the overall POF due to a distribution, $m$, of flaws is:

$$
F(\boldsymbol{m})=1-\prod_{\mathrm{r}=1}^{R}\left(1-f_{r}\right)^{m_{r}}
$$

The mean POF is calculated using the flaw population PDF as follows:

$$
\overline{F_{n}}=\sum_{m_{1}} \sum_{m_{2}} \ldots \sum_{m_{R}} F(\boldsymbol{m}) P_{M \mid N}(\boldsymbol{m} \mid \boldsymbol{n})
$$

Where the summation ranges are as previously described.

The computational requirements of equation (6) deserve some discussion. The summations in equation (6) and in the denominator term, $P_{N}$, in equation (4) are potentially very costly to calculate. Two considerations reduce the computational requirements considerably:

1. When flaws in adjacent flaw size range bins result in identical values for the POF, $f_{i}$, the adjacent bins can be combined, reducing the dimensions of the problem. Typically, the smallest flaws, which fall into the first few size range bins, result in zero probability of failure. These bins can be combined. Similarly, a few bins containing the largest flaws can sometimes be combined when flaws in these bins result in certain failure (POF=1).

2. The magnitude of the PDF given by equation (4) is usually only significant over a small region of $R$-dimensional space. The summations only need to be done over this region. This region can be determined beforehand by first finding the 
distribution, $m$, corresponding to the maximum value of the PDF and then determining the range of values for each $m_{i}$ that result in significant magnitudes for the PDF.

\section{FRACTURE MECHANICS}

The essential element of the fracture mechanics model is an empirical equation relating the Mode I (tensile loading) stress intensity factor, $K_{I}$, to the applied stress, $S_{t}$. This equation, derived by Newman and Raju for semi-elliptical surface cracks in a plate loaded in combined bending and tension, is

$$
K_{I}=S_{t} \sqrt{\frac{\pi a}{Q}} F
$$

where $a$ is crack depth, $Q$ is the shape factor, and $F$ is the boundary correction factor. The shape factor, $Q$, is given by

$$
Q=1+1.464\left(\frac{a}{c}\right)^{1.65}
$$

where $c$ is the half-length of the surface crack. The boundary correction factor, $F$, is given by

$$
F=\left[M_{1}+M_{2}\left(\frac{a}{t}\right)^{2}+M_{3}\left(\frac{a}{t}\right)^{4}\right] f_{\phi} g f_{w}
$$

where $t$ is the thickness of the plate and $\phi$ is the parametric angle of the ellipse. Other terms appearing in the above equation are as follows:

$$
\begin{gathered}
M_{1}=1.13-0.09\left(\frac{a}{c}\right) \\
M_{2}=-0.54+\frac{0.89}{0.2+\frac{a}{c}} \\
M_{3}=0.5-\frac{1.0}{0.65+\frac{a}{c}}+14\left(1.0-\frac{a}{c}\right)^{24} \\
g=1+\left[0.1+0.35\left(\frac{a}{t}\right)^{2}\right](1-\sin \phi)^{2} \\
f_{\phi}=\left[\left(\frac{a}{c}\right)^{2} \cos ^{2} \phi+\sin ^{2} \phi\right]^{\frac{1}{4}}
\end{gathered}
$$




$$
f_{w}=\left[\sec \left(\frac{\pi c}{2 b} \sqrt{\frac{a}{t}}\right)\right]^{\frac{1}{2}}
$$

where $b$ is the half-width of the cracked plate.

The probability of failure is, by definition, the probability that the stress intensity factor exceeds some critical value, i.e.

$$
\operatorname{Prob}\left\{K_{I}>h K_{I c}\right\}
$$

where $K_{i c}$ is the fracture toughness and $h$ is a correction factor determined empirically. For this work $h$ was assumed to be 1.15. Values for the fracture toughness were taken from the literature.

The applied stress, $S_{t}$ in equation (7), was considered to be a random variable with a gaussian PDF. The stress intensity factor, $K_{I}$, is therefore a gaussian random variable also. The POF for a single crack is determined by first calculating the mean and standard deviation of the stress intensity factor from the mean and standard deviation of the applied stress using the above fracture mechanics equations. The area under the stress intensity factor PDF that exceeds the critical threshold, $h K_{I c}$, is by definition the POF.

For this work all cracks are considered to have the same aspect ratio, so crack depth is the only size parameter of interest. The depth of a crack assigned to a depth range bin is assumed be equal to the midpoint of the depth range for that bin. Using this crack depth and the method outlined in the previous paragraph, a POF for each flaw size bin is calculated. For the $i$-th bin this POF is the parameter $f_{i}$ appearing in equation (5).

\section{PROBABILITY OF DETECTION AND CRACK SIZE DISTRIBUTION}

In order to apply this failure analysis methodology to a structure the relationship between the POD and crack depth must be estimated. For this work we assume the following form for the POD function: ${ }^{5,6}$

$$
P_{D}(A)=1-\varepsilon-\frac{1}{2}(1-\varepsilon) \operatorname{erfc}\left(v \ln \left(\frac{A}{A^{*}}\right)\right)
$$

where $P_{D}$ is the POD, $A$ is the area of the crack, $A^{*}$ is the crack area corresponding to $P_{D}=50 \%, \varepsilon$ is the smallest possible probability of nondetection for very large cracks, and $v$ is an empirically determined "slope."

Khaleel and Simonen, ${ }^{6}$ at Pacific Northwest Laboratory (PNL), have identified three POD curves corresponding to three levels of NDE reliability. These NDE levels are described as follows:

1. Marginal performance: In the judgment of the PNL experts, a POD performance that is described by this curve would represent a team having only a small chance of passing an Appendix VIII performance demonstration.

2. Very good performance: In the judgment of the PNL experts, this curve corresponds to a team that significantly exceeds the minimum level of performance needed to pass an Appendix VIII performance demonstration test.

3. Advanced performance: In the judgment of the PNL experts, this curve corresponds to a team that has a level of performance significantly better than expected from present day teams that have passed an Appendix VIII-type of performance demonstration. Such a team would need to apply advanced technologies and/or improved procedures that could be developed in the future.

Table 1 presents the POD curve parameters for the three levels of NDE reliability used by Khaleel and Simonen. These three POD curves were used for this work. They are plotted in figure 1. 
We assume an exponential form for crack depth distributions. Figure 2 shows the exponential distribution of 476 cracks in 40 crack depth bins. Figures 3 and 4 show distributions in which 234 and 118 cracks were apportioned according to the exponential crack depth distribution used in figure 2. Figures 5, 6, and 7 show distributions of detected cracks, consistent with the presence of 476 cracks, as in figure 2 , and the POD curves defined in table 1 . We conducted test cases using these three distributions of detected cracks and six more distributions consistent with figures 3 and 4 and table 1 .

\section{PROBABILITY OF FAILURE CALCULATIONS}

Nine test cases are presented here. Each test case results from one of the three POD curves described above and one value for the total number of cracks actually present. The assumed fracture mechanics parameters are as follows:

- plate thickness

- parametric angle of the elliptical cracks

- aspect ratio of cracks

- width of plate

- mean applied stress

- percent standard deviation of applied stress
$14.3 \mathrm{~mm}$
$90^{\circ}$
4.5
$6.096 \mathrm{~m}$
$186.2 \mathrm{~Pa}$
$10 \%$

Figure 8 shows the POF due to one crack in each of 40 crack depth bins, calculated assuming the above fracture mechanics parameters. Equations, (4), (5), and (6) are used to calculate the overall POF of the structure from the POF curve in figure 8 and the crack population PDF (equation (4)). The results of the nine test cases are summarized in table 2.

The benefit of more reliable NDE is clear from table 2. As NDE becomes more reliable the calculated POF of the structure approaches the POF that would be calculated if the actual crack population were known. As NDE becomes less reliable the probability of larger undetected cracks becomes greater and the calculated POF increases.

The benefit of improved NDE seems to increase when fewer cracks are present. This is because the depths of the largest cracks decrease as the actual crack population decreases. These largest cracks influence the POF calculations the most, especially when more reliable NDE techniques are used.

\section{DISCUSSION}

In many risk assessment models a crack population is assumed for a structure without NDE input. Such assumptions are problematic for a number of reasons, one being the difficulty of predicting changes in the crack population in a component after years of service. This prediction task requires extensive experience with the materials used to fabricate the structure and with the structure's operating environment in order to identify the processes for crack initiation and growth. The luxury of such experience does not exist generally because of the operational realities of most industries. As a result, substantial conservatism exists in models predicting structural integrity and lifetime extension. The roll of NDE in lifetime prediction has generally been overlooked by these conservative prediction models. This work has demonstrated that NDE can play a significant roll in lifetime prediction.

The probabilistic model presented here is useful for quantifying the improvements in reliability resulting from NDE, and studying the effects of different NDE performance levels. The probabilistic fracture mechanics model described here is quite simple, considering variability only in the applied stress. However, the methodology described in this paper could be used for more complicated models in which variability and uncertainty in several parameters are considered. Such a model would permit the simultaneous study of the effects of NDE and variability and uncertainty in material properties, loading conditions, and flaw characteristics.

This study suggest that NDE can play a significant role in reducing the probability of failure of structures if the inspection procedures satisfy strict minimum performance requirements. The application of this methodology could provide justification for extending the lifetimes of pressure vessels or other industrial structures. 


\section{ACKNOWLEDGEMENTS}

This work was supported through the INEEL Laboratory Directed Research and Development Program under DOE Idaho Operations Office Contract DE-AC07-94ID13223.

The authors are employed by Lockheed Martin Idaho Technologies Company, P. O. Box 1625, Idaho Falls, ID 83415-2209.

\section{REFERENCES}

1. S. A. Chavez, C. L. Atwood, P. G. Ellison, and J. L. Jones, "Estimating structural failure frequency of degraded steam generator tubes," Proc. Of the $4^{\text {th }}$ International Conference on Nuclear Engineering, Mar. 10-14, 1996, New Orleans, LA, USA, pp. 411-426, American Society of Mechanical Engineers, New York, NY, USA, 1996.

2. J. H. Phillips, W. S. Roesener, H. L. Magleby, W. Reuter, and V. Geidel, Development and Demonstration of Methods to Include Aging of Passive Components in PRAs, NUREG/CR-5730, Nuclear Regulatory Commission, Washington, D.C., 1991.

3. O. J. V. Chapman, "Simulation of defects in weld construction," Reliability and Risk in Pressure Vessels and Piping, PVP-Vol. 251, pp. 81-89, American Society of Mechanical Engineers, New York, 1993.

4. J. C. Newman, and I. S. Raju, "Analysis of surface cracks in finite plates under tension and bending loads," NASA Technical Paper 1578, National Aeronautics and Space Administration, Washing, DC, December 1979.

5. D. O. Harris, D. D. Dedhia, and S. C. Lu, Theoretical and User's Manual for pc-PRAISE, A Probabilistic Fracture Mechanics Computer Code for Pipe Reliability Analysis, NUREG/CR-5864, UCRL-ID-109798, U.S. Nuclear Regulatory Commission, Washington, D.C.

6. M. A. Khaleel, and F. A. Simonen, "The effects of initial flaw sizes and inservice inspection on piping reliability," 1994 ASME Pressure Vessel and Piping Conference, Minneapolis, Minnesota, June 19-23, 1994, PVP-Vol. 288, pp. 95-107, American Society of Mechanical Engineers, 1994. 


\begin{tabular}{|l|c|c|c|}
\hline $\begin{array}{c}\text { Inspection } \\
\text { Performance }\end{array}$ & $\begin{array}{c}\text { Crack depth } \\
\text { for 50\% POD }\end{array}$ & $\epsilon$ & $\nu$ \\
\hline Marginal & .40 & 0.10 & 1.6 \\
\hline Very good & .15 & 0.02 & 1.6 \\
\hline Advanced & .05 & 0.005 & 1.6 \\
\hline
\end{tabular}

Table 1. POD curve parameters for three NDE reliability levels.

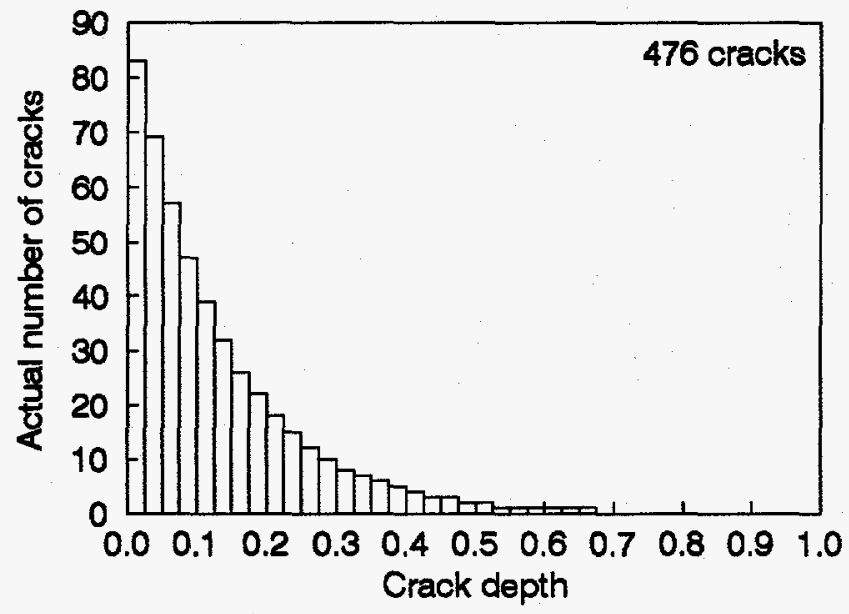

Figure 2. Exponential distribution of 476 cracks in 40 crack depth bins.

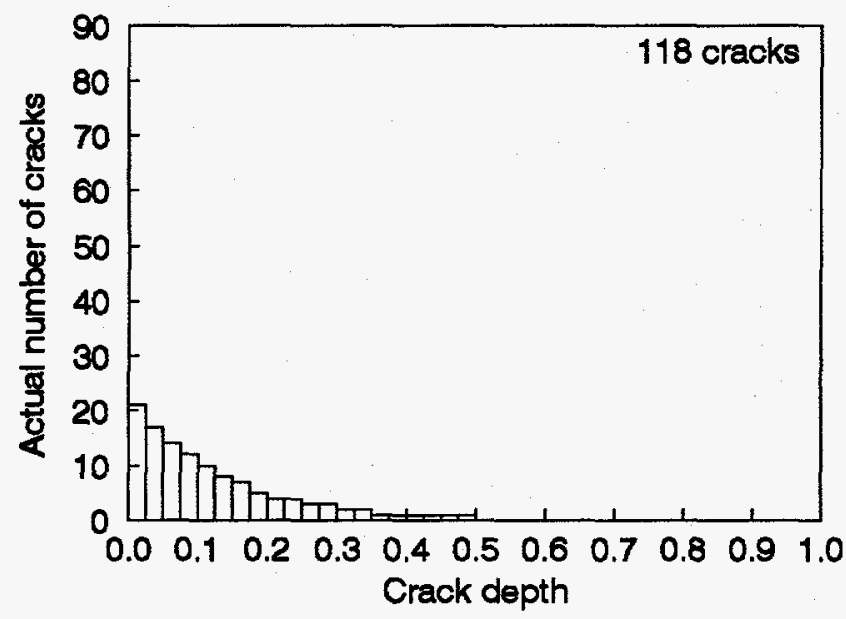

Figure 4. Exponential distribution of 118 cracks in 40 crack depth bins.

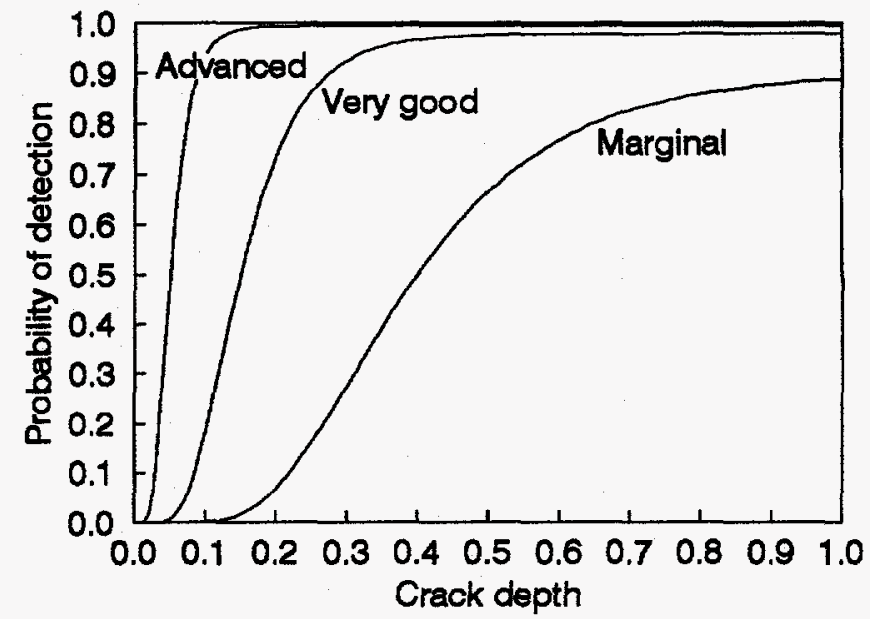

Figure 1. POD curves for three levels of NDE reliability.

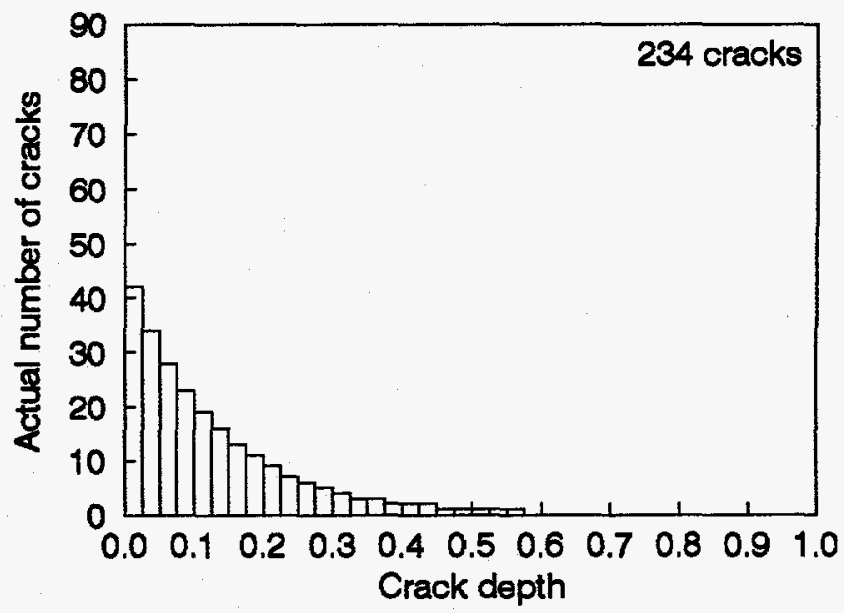

Figure 3. Exponential distribution of 234 cracks in 40 crack depth bins.

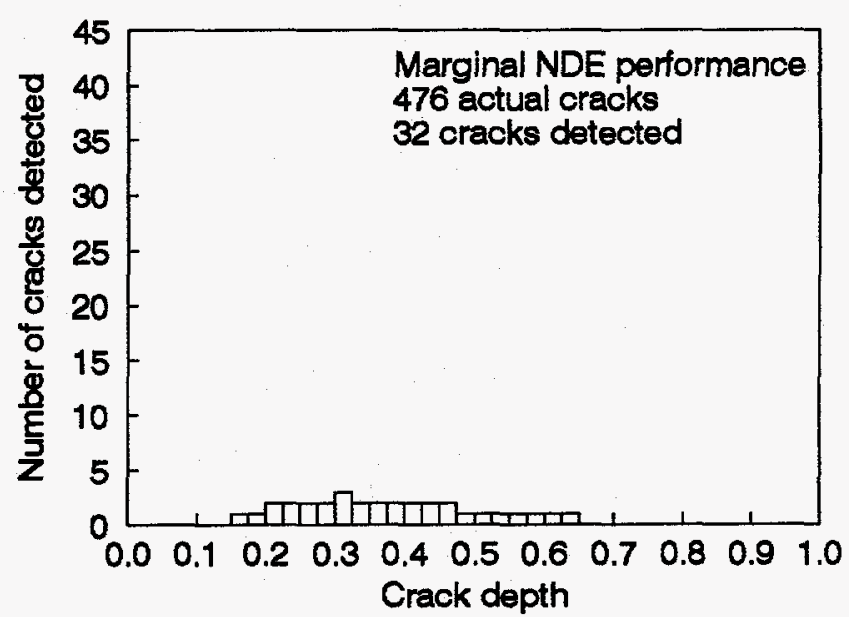

Figure 5. Number of cracks detected when NDE performance is marginal. 


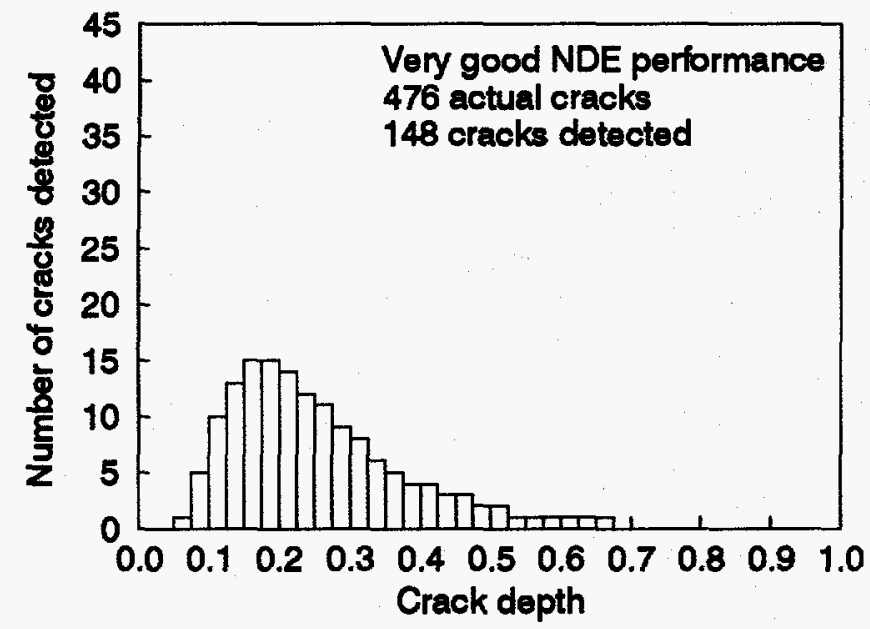

Figure 6. Number of cracks detected when NDE performance is very good.

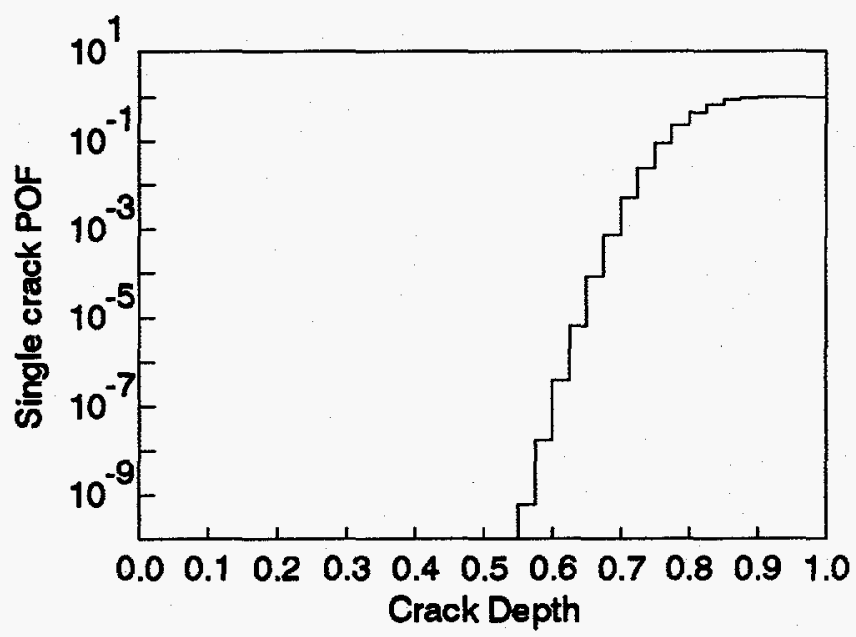

Figure 8. POF due to a single crack in each crack depth bin.

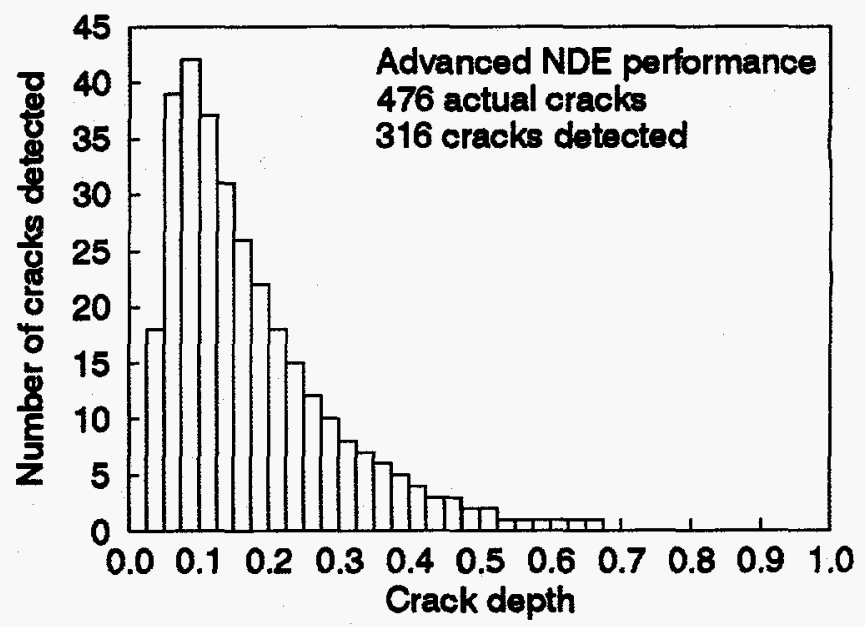

Figure 7. Number of cracks detected when NDE performance is advanced.

\begin{tabular}{|l|c|c|c|}
\hline $\begin{array}{c}\text { Inspection } \\
\text { Performance }\end{array}$ & $\begin{array}{c}476 \text { actual } \\
\text { cracks }\end{array}$ & $\begin{array}{c}234 \text { actual } \\
\text { cracks }\end{array}$ & $\begin{array}{c}118 \text { actual } \\
\text { cracks }\end{array}$ \\
\hline Marginal & $8.0 \times 10^{-2}$ & $4.0 \times 10^{-2}$ & $2.0 \times 10^{-2}$ \\
\hline Very good & $1.3 \times 10^{-2}$ & $3.5 \times 10^{-3}$ & $3.9 \times 10^{-4}$ \\
\hline Advanced & $4.9 \times 10^{-4}$ & $1.2 \times 10^{-6}$ & $8.2 \times 10^{-8}$ \\
\hline
\end{tabular}

Table 2. POF calculations for three different NDE reliability levels and three crack populations. 